\title{
Clinical and serological study of human alveolar echinococcosis in Slovakia in relation to the outcome of chemotherapy
}

\author{
J. KINČEKOVÁ ${ }^{1}$, G. HRČKOVÁ ${ }^{1}$, H. AUER ${ }^{2}$, M. SZILÁGYIOVÁ ${ }^{3}$, J. HUDAČKOVÁ ${ }^{4}$, M. STANISLAYOVÁ \\ H. POLÁČEK ${ }^{6}$, K. ŠIMEKOVÁ ${ }^{3}$
}

\begin{abstract}
${ }^{1}$ Parasitological Institute of the Slovak Academy of Sciences, Hlinkova 3, 04001 Košice, Slovak Republic; E-mail: dr.kincek@zoznam.sk; ${ }^{2}$ Clinic of Infectious Diseases of Jessenius Medical Faculty of Komensky University and University Hospital of Martin, Slovak Republic; ${ }^{3}$ Dept. of Medical Parasitology, Clinical Institute of Hygiene and Medical Microbiology, Medical University of Vienna, Austria; ${ }^{4}$ Infectious Dept. of J. A. Reiman University Hospital of Prešov, Slovak Republic; ${ }^{5}$ Radiodiagnostic Dept. of J. A. Reiman University Hospital of Prešov, Slovak Republic;

${ }^{6}$ Clinic of Radiology Diseases of Jessenius Medical Faculty of Komensky University and University Hospital of Martin, Slovak Republic
\end{abstract}

\section{Summary}

Alveolar echinococcosis (AE), caused by proliferating metacestodes of the parasitic fox tapeworm Echinococcus multilocularis, is a life-threatening disease in humans. In this study we report four human cases of AE in Slovak Republic with regard to various clinical manifestations and susceptibility to chemotherapy with albendazole or mebendazole. Patients were monitored serologically by ELISA test and Western Blots within $2-5$ years after initiation of chemotherapy/surgery. Using computerized tomography (CT) we compared morphological changes of the parasitic lesions in the liver during the course of treatment.

The parasitic lesions in the CT were manifested as nodular hyperplasia with hyper or hypodense zones and calcified foci within the lesion. In other cases the lesion was visualised as a hypodense poorly-delineated septated focus without calcifications. In the last case, the diagnosis of AE was confirmed only at surgery. Calcified foci found inside the main parasitic lesions were the only abnormality that was detected in two cases and were absent in other two cases. The levels of specific anti-parasitic antibodies followed the pathomorphological changes in the livers. Total IgG levels to Em2+ and EmP antigen declined gradually during the follow-up ( $1-5$ years) and disappeared only in the patient receiving radical surgery (cured). In comparison with total IgG, concentration of the IgG4 antibody subclass seemed to correlate more adequately with the outcome of therapy as their levels decreased in improved/stabilised patients, but were elevated in "aggravated" patients. We showed that, in patients with AE in Slovakia, radical surgery of parasitic foci proved to be the most successful treatment and, in inoperable cases, albendazole and mebendazole were differently effective. Moreover, IgG4 levels rather than total IgG to protoscoleces antigens proved to be more sensitive serological marker of the progress of therapy.
Key words: Echinococcus multilocularis; CT imaging; chemotherapy; serology

\section{Introduction}

Alveolar echinococcosis (AE) is a parasitic disease caused by Echinococcus multilocularis metacestodes and primaryly affects the liver and may infiltrate neighbouring organs. Cells frequently detach and disseminate via lymph nodes and blood vessels, thus leading to metastasis in a large parenchymatous organs. Therefore, AE is often compared with a slow growing liver cancer (Craig, 2003). Lethality of natural AE reaches $94-100 \%$ within $10-15$ years after diagnosis in untreated or inadequately treated human patients (Amman \& Eckert, 1996). The natural life cycle of E. multilocularis in Europe involves predominantly red foxes as the definitive hosts, less commonly dogs or cats, while rodents and humans act as the intermediate hosts. Foxes are infected by eating infested rodents. The mature segments of the parasite, containing eggs, are dispersed in the environment via the faeces of carnivores. The eggs may contaminate various types of food, including fruits and vegetables collected from gardens or infected meadows and drinking water. Humans become infected by ingestion of contaminated food and water. The infection may become symptomatic only after 5-10 years (BressonHadni et al., 2000). The typical age at onset is between 50 -70 , and the infection is less often at younger age.

In some endemic regions in Central Europe and Japan, the prevalence ranges from 2 to 40 cases per 100000 inhabitants (Eckert \& Deplazes, 1999). During 5 years (19962000), 10 new cases were reported in Austria, 48 in Germany and 16 in Poland (Kern et al., 2003). The highest published incidence was reported from eastern France with 
a ratio of 152/100 000, but this study included cases also with inactive lesions and it concentrated on farmers, a recognised risk group (Bresson-Hadni et al., 2000).

At present, many authors believe that the rising occurrence of $\mathrm{AE}$ in humans is probably due to the overpopulation of red foxes (Lucius \& Bilger, 1995; Giraudoux et al., 2001), as the result of successful oral vaccination against rabies. Since 2000, 6 cases of AE have been diagnosed in Slovakia. First two cases (Kinčeková et al., 2001; 2002) were found in 70-year-old women and two other cases were in men over sixty. The last two infections were in young humans (30- and 13-years old). Diagnosis of AE is usually based on history, clinical signs, morphological changes identified on imagining studies (US, CT), and then is confirmed histologically and serologically. In the primary diagnosis of AE, the US and CT scans are only supplementary. CT can better visualise lesions and newly formed foci, US is more suitable for visualising necrosis and abscesses (Stanley, 1983; Hattinger et al., 1996). Albendazole and mebendazole are drugs of choice, recommended for the treatment of both cystic (CE) and alveolar echinococcosis (AE), whereas in case of AE, these drugs have primarily parasitostatic rather than parasitocidal effect (Eckert \& Amman, 1995). The overall success rate of these drugs ranges between 55 and $97 \%$ and depends on the stage of infection and susceptibility to chemotherapy. Therefore in inoperable cases a high incidence of relapses in the liver lesions of up to $25 \%$ occurs (Ishizu et al., 1997). Total IgG specific antibodies often persist in sera of patients for a long time, even after recovery, and therefore provide only complementary data about the progress in therapy ( $\mathrm{Ri}-$ gano et al., 1995). In chronic helmith infections IgG1 and IgG4 antibody subclasses contribute by a substantial part to the total specific IgG antibodies. In patients with alveolar echinococcosis, during their follow up to surgery and /or chemotherapy, Wen et al. (1995) found that the levels of specific IgG4 antibody isotype correlated with active/ inactive disease and declined markedly in cured patients. In this study we present 4 cases of $\mathrm{AE}$ that were diagnosed in Slovakia between the years $2000-2005$. All patients were elderly with similar epidemiological history and AE was confirmed incidentally at the late stage. The aim of this study was to compare the course of infection (regression, stabilisation, progression) obtained by means of CT methods with the changes in specific IgG and IgG4 antibody levels to E. multilocularis commercial Em2+ antigen and somatic antigens from protoscoleces (EmP).

\section{Patients and Methods}

\section{Patients}

Since 2000, 4 cases of AE have been diagnosed. Two cases in two 70-year-old females and two cases in 69- and 77year-old males. The infection risks have resulted from their epidemiological histories. The first patient had worked for 20 years as a forest worker, consuming forest fruits. Other patients lived in the houses with a fruit and vegetable garden in the close vicinity of a forest. In all cases, a primary liver infection was presented. Patients originated from the northern and eastern regions of Slovakia and were admitted into the local hospitals. Their follow-up was performed at University hospitals in that region. CT (Computer tomography) was performed using CT scanner Somatom Sensation 10 (Siemens), rotation time of $0.5 \mathrm{~s}$ and multislice data acquisition with UFC ${ }^{(\mathrm{tm})}$ detector up to 10 slices simultaneously. The patients were prepared by ingestion of Telebrix and scanning was performed before and after intravenous administration of bolus contrast medium (100 ml Optiray 350, Healthcare Deutschland GmH).

\section{Antigens and serological tests}

For the primary screening of specific parasitic antibodies in patients, an indirect ELISA test was performed using Commercial ELISA KIT Echinococcus multilocularis $\mathrm{Em}^{+}$antigen (Bordier affinity products SA, Switzerland) according to the manufacturer's instructions. The diagnosis of $\mathrm{AE}$ was confirmed using Echinococcus Western Blot IgG Kit (LDBIO Diagnostics, Lyon, France) by reading of the specific bands between 7 and $28 \mathrm{kDa}$. For determination of specific IgG4 antibody isotype, somatic antigen from isolated E. multilocularis protoscoles (EmP) was prepared as follows. Parasitic cysts were recovered from the peritoneal cavity of infected gerbils (Meriones unguiculatus) and were placed into RPMI 1640 medium containing antiobitics. After the mincing the cysts, the tissue was passed through a Cell Dissociation Sieve Tissue Grinder Kit, so as to obtain a culture of protoscoleces. Culture medium contained the protease inhibitors $1 \mathrm{mM}$ phenylmethylsulphonyl fluoride (PMSF), $0.01 \mathrm{mM}$ leupeptin, $0.05 \mathrm{mM}$ pepstatin and $1 \mathrm{mM}$ phenanthroline (all from Sigma). Protoscoleces were washed in PBS ( $\mathrm{pH}$ 7.2) and sonicated at $4^{\circ} \mathrm{C}$ until no intact specimen were detected by microscopy. The resulting suspension was centrifuged at $10000 \mathrm{~g}$ for $30 \mathrm{~min}$ at $4^{\circ} \mathrm{C}$ and supernatant containing EmP antigen was collected, aliquoted and stored at $-70^{\circ} \mathrm{C}$. Concentration of proteins was determined by the Bradford protein assay (Bio-Rad, UK) and in ELISA tests the concentration of protein was $3 \mu \mathrm{g} / \mathrm{ml}$.

Patients' sera were used in dilution of $1: 100$ in $4 \%$ fat-free milk in PBS (pH 7.2) in tests using Em $2^{+}$E.m. kit as well EmP antigen. Secondary antibodies for detection of total IgG with EmP antigen were HRP-conjugated goat antihuman IgG $\gamma$-chain, affinity purified (Sigma) in a dilution of 1:6000. Levels of IgG4 specific antibody subclass was determined using HRP-conjugated mouse anti-human IgG4, monoclonal (ICN, USA) in dilution 1:8000. The substrate solution contained $0.04 \%$ of $o$-phenylenediamine (OPD) (Sigma) and $0.02 \% \mathrm{H}_{2} \mathrm{O}_{2}$ in citric phosphate buffer (pH 4.5). Absorbance values were measured at $492 \mathrm{~nm}$ on the plate reader (Multiscan PLUS; Labsystem). Three positive $\mathrm{AE}$ sera and two from the healthy donors were used as standard controls. The standard control values for IgG and IgG4 to EmP antigen were the highest ratios between the positive and negative controls. Finally, the recorded OD values were used to calculate antibody units (AU) as the percentage of the positive standard control values accord- 
ing to Gottstein et al. (1989). Levels of specific IgG and IgG4 antibodies obtained with EmP antigen are presented in Table 1 .

Biochemical, haematological and histological (stained with hematoxylin-eosine, alcian blue, PAS) examinations of the patients were performed by the routine laboratory methods.
Case 2. A 75-year old female

The patient had been treated for 10 year for anaemia of unknown origin. At check-up in 2000, haematological findings significantly progressed. Abdominal ultrasound showed a mass in the right liver lobe. The lobbed delineated non-homogenous focus (size $14 \times 17 \mathrm{~cm}$ ) containing

Table 1. Serological analysis of total IgG and IgG4 specific antibody levels to EmP antigen in 4 patients with alveolar echinococcosis before therapy and during follow-up to 5 years

\begin{tabular}{|c|c|c|c|c|c|c|c|c|}
\hline \multirow{3}{*}{$\begin{array}{c}\text { Years } \\
\text { after } \\
\text { therapy }\end{array}$} & \multicolumn{8}{|c|}{ ELISA results expressed in antibody units $(\%) *$} \\
\hline & \multicolumn{2}{|c|}{ CASE 1} & \multicolumn{2}{|c|}{ CASE 2} & \multicolumn{2}{|c|}{ CASE 3} & \multicolumn{2}{|c|}{ CASE 4} \\
\hline & IgG & IgG4 & $\operatorname{IgG}$ & IgG4 & IgG & IgG4 & IgG & IgG4 \\
\hline 0 & 98 & 28 & 70 & 58 & 69 & 39 & 135 & 75 \\
\hline $1 / 2$ & 87 & 37 & 86 & 38 & 47 & 51 & 97 & 61 \\
\hline 1 & 69 & 34 & 83 & 45 & 41 & 12 & 86 & 80 \\
\hline 2 & 50 & 22 & 89 & 62 & 26 & 0 & 76 & 56 \\
\hline 3 & 17 & 19 & 63 & 87 & 0 & 0 & 72 & 61 \\
\hline 4 & ND & ND & 60 & 98 & 0 & 0 & 23 & 40 \\
\hline 5 & & & 52 & 95 & & & ND & ND \\
\hline
\end{tabular}

*ELISA results are shown as antibody units expressed as a percentage of the positive standard control value (see Methods); ND = not determined because no serum was available

\section{Case reports}

Case 1. A 75-year old female

The patient started to suffer from dyspepsia, accompanied with right epigastric pain in 1994. No diagnosis had been established. In September 1999, she was admitted to the hospital with biliary colic. The US visualised cholecystolithiasis and foci in the right liver lobe. CT scan (Fig. 1a) confirmed a non-homogenous mass, approximately $10 \times 8$ $\mathrm{cm}$ in size, with calcifications. Scintigraphic examination of the liver did not suggest the metastases or hepatocelular carcinoma. Open liver biopsy and cholecystektomy was performed. Histopathological examination of excised tissue sections after staining with PAS, indicated on E. multilocularis infection. In this foci CT showed presence of calcifications. After two years of albendazole therapy $(2 \mathrm{x}$ $400 \mathrm{mg} /$ day), the infection foci have regressed approximatelly $50 \%$ (Fig. 1b). Laboratory findings performed before chemotherapy revealed anaemia (Haemoglobin 100 $\mathrm{g} / \mathrm{l})$, high erythrocyte sedimentation rates (ESR $63 \mathrm{~mm} / \mathrm{h}$ ) and the increase in alkaline phosphatase $(3.81 \mu \mathrm{kat} / \mathrm{l})$. Eosinophilia was not detected. Within the course of therapy, the normalization of laboratory parameters occurred. The levels of specific total IgG antibody before initiation of albendazole therapy were high $(91 \%)$ and declined markedly to a low level within three years of follow-up (17\%) (Table 1). The banding pattern on commercial strip corresponded to that indicated for $\mathrm{AE}$ with the most intense specific band of $26-28 \mathrm{kDa}$ and low intense bands of 16 and $18 \mathrm{kDa}$. Specific E. granulosus band with MW 7 kDa was not visible. IgG4 antibody response to EmP antigen had increased following initiation of chemotherapy within the first half of year $(37 \%)$ of follow-up and remained elevated $(19 \%)$ within the period of examination (3 years). dense and hyper-dense zones in the $7-8$ liver segment was seen on CT scans (Fig. 2a). Numerous calcifications were present within this lesion. Laparoscopic biopsy followed by histology and specific PAS staining of sections detected the fragments of parasite membrane and the signs of malignancy were not confirmed. Laboratory findings performed before chemotherapy revealed anaemia (Hemoglobin $115 \mathrm{~g} / \mathrm{l}$ ), high erythrocyte sedimentation rates (ESR $68 \mathrm{~mm} / \mathrm{h})$ and the increase in alkaline phosphatase $(4.85$ $\mu \mathrm{kat} / \mathrm{l})$. Eosinophilia was not detected. Albendazole therapy $(2 \times 400 \mathrm{mg} /$ day) was initiated after 3 months, but drug administration had to be stopped due to the patient intolerance of drug and deterioration of selected biochemical parameters (SGPT - $2.28 \mu \mathrm{kat} / \mathrm{l}$; SGOT - 0.96 $\mu \mathrm{kat} / \mathrm{l})$. The change to mebendazole (1000 mg/day) has not, even after 4 years, affected the size and appearance of the foci (Fig. 2b). Total IgG antibodies were relatively high (see Table 1) during the whole period of treatment with mebendazole. Serum levels of IgG4 were markedly elevated at first diagnosis of AE $(58 \%)$ following by some decline after short-term of ABZ treatment $(38 \%)$. Then the gradual increase of IgG4 was seen up to 5 years of followup $(95 \%)$ indicating on activation of parasitic lessions and possible relapse of disease. Western Blot banding pattern before initiation of therapy showed the presence of the most intense $26-28 \mathrm{kDa}$ specific band and less intense echinococcus-specific bands.

Case 3. A 62-year old male

The patient was previously treated for ischemic heart disease, arterial hypertension and bronchial asthma. He also suffered from prostate hyperplasia. Examination revealed liver enlargement and pains on palpation in the central and 

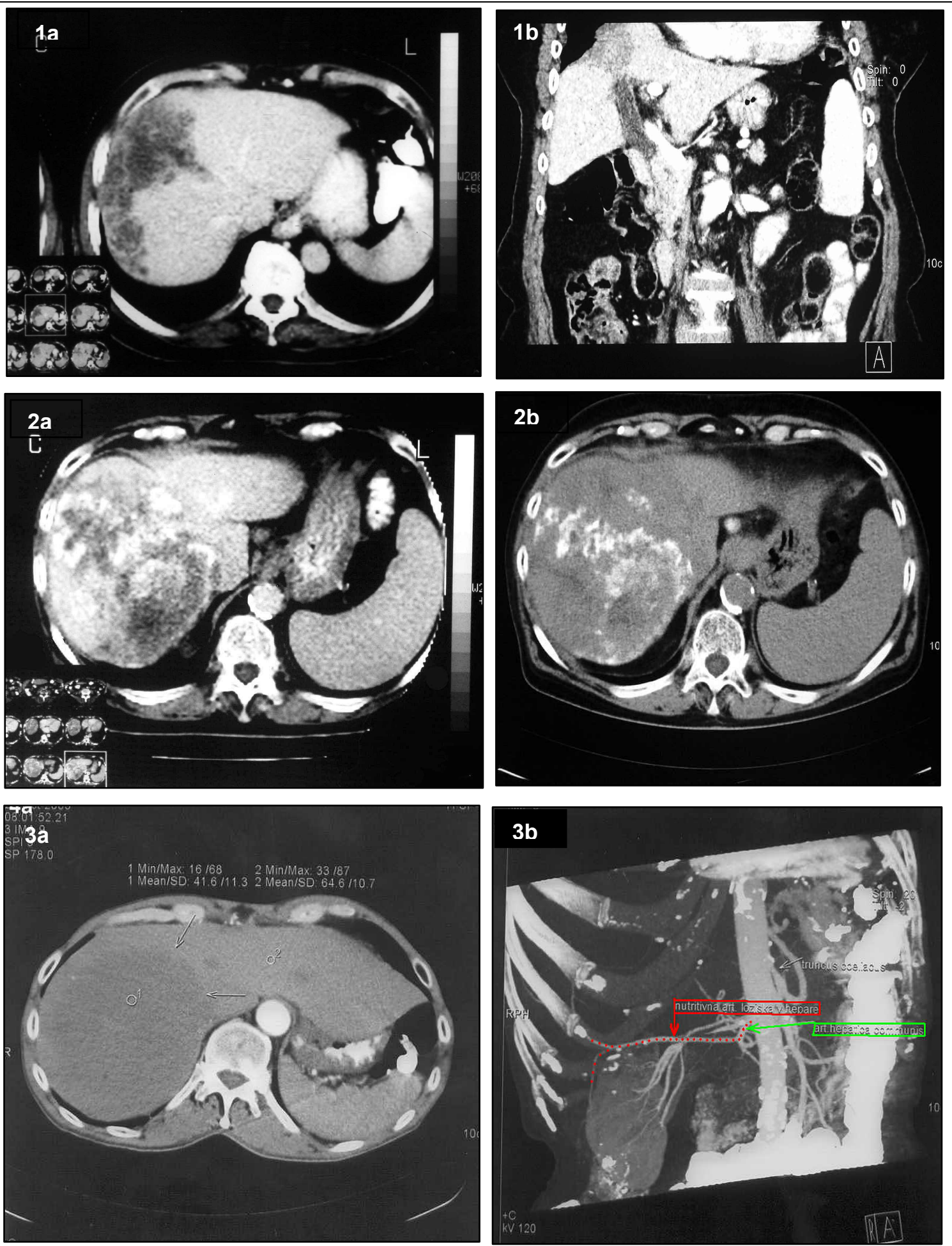

Fig. $1 \mathrm{a}-\mathrm{b}$ (Case 1), 1a-CT immage of right lobe before therapy showing nodular hyperplazia of hepatal parenchyma with hypodense or cystoide zones with numerous microcalcifications in the lesions. $1 \mathrm{~b}-\mathrm{CT}$ findings after two-years of therapy with albendazole.

Fig. $2 \mathrm{a}-\mathrm{b}$ (Case 2): $2 \mathrm{a}-\mathrm{CT}$ scans showing non-homogenous lesion containing dense and hyperdense cysts in the $7-8$ right liver segment. $1 \mathrm{~b}-$ situation after four years of chemotherapy with mebendazole.

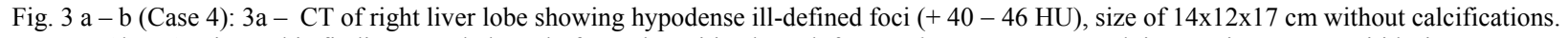
$3 \mathrm{~b}$ - Angiographic finding revealed newly formed nutritive branch from $a$. hepatica com. supplying nutrients to parasiti lesion.

right epigastrium. Laboratory parameters moderately increased similarly as in case 1 and 2 . The patient complained of periodic pain and weight loss of $10 \mathrm{~kg}$ during 9 216 months. The CT scan showed hypodense foci in the left liver lobe, $6.4 \times 5 \mathrm{~cm}$ in size, irregularly shaped with the necrotic cavity in the centre. Pathological examination of 
the liver was negative for malignancy. Radical surgery of the whole lobe (hemihepatectomy) was performed. The diagnosis of $\mathrm{AE}$ was indicated firstly after pathological examination of the removed liver lobe showing PAS positive reaction for parasitic tissue. Serological examination for presence of total $\mathrm{IgG}$ antibodies to $\mathrm{Em} 2+$ and $\mathrm{EmP}$ antigen confirmed AE (69\%). The patient has been on albendazole treatment $(2 \times 400 \mathrm{mg} /$ day $)$ for more than one year, which resulted in the marked decline of total IgG (see Table). IgG4 antibody levels also declined, more rapidly than total IgG within two years of follow-up. Post-operative recovery uneventful. Western Blot examination of sera taken before surgery confirmed infection with E. multilocularis and the patient's serum reacted with bands of MW $26-28 \mathrm{kDa}$ and $16 \mathrm{kDa}$.

\section{Case 4. A 77-year old male}

The patient had 8 years' history of ischemic heart disease and angina pectoris. In 2002 he suffered from a complicated influenza, with a weight loss of $6 \mathrm{~kg}$. He was admitted to a cardiology ward in October 2003. During his hospitalisation the US of the liver was performed, revealing tumorous process in the liver and numerous cholecystolithiasis. CT scan (Fig. 3a) visualised expansive foci in the right liver lobe, $14 \times 12 \times 17 \mathrm{~cm}$ in the size. Calcifications in the foci were not detected. The foci itself $(+40$ to +46 $\mathrm{HU}$ ) did not appear to change in the post-contrast CT scans - either in arterial or venous phase. In this case, angiography of hepatic arteries showed newly formed nutritive branch from $a$. hepatica com., supplying nutrients to the foci (Fig. 3b). Histological and serological examination (ELISA, WB) confirmed alveolar echinococcosis showing very high levels of IgG antibodies (135\%) (Table 1). Laboratory findings performed before chemotherapy revealed anaemia (Hemoglobin $115 \mathrm{~g} / \mathrm{l}$ ), high erythrocyte sedimenttation rates (ESR $147 \mathrm{~mm} / \mathrm{h}$ ) and the increase in alkaline phosphatase (5.06 $\mu \mathrm{kat} / \mathrm{l})$, while aminotransferases (SGPT and SGOT) were increased only moderately. Eosinophilia was not detected. Toxicity of one year-lasting albendazole therapy ( 2 x $400 \mathrm{mg}$ /day) was significant (fever, nausea, abdominal pains). Moreover, CT examinations revealed the occurrence of three new abscess foci inside the parasitic cyst $(1.5-2.5 \mathrm{~cm}$ in size $)$, density of $+16 \mathrm{HU}-+19 \mathrm{HU}$ with colliquation. The surgical evacuation of purulent content of these abscess foci was performed. In this case, even after three years of albendazole therapy, total IgG antibodies did not decline considerably. On WB examination, intense specific bands of $26-28 \mathrm{kDa}$ and less intense bands of MW of 18 and $16 \mathrm{kDa}$ were detected (not shown). Although IgG4 levels were lower than IgG during chemotherapy, IgG4 antibody activity did not have similar and consistent declining pattern and remained higher at the last year (4) of examination (40\%).

\section{Discussion}

In this study we reported about four patients in Slovakia with $\mathrm{AE}$, which differed in the localisation and extent of parasitic lesions in the liver, in serological parameters and the susceptibility to albendazole/mebendazole chemotherapy. Search for reliable markers indicating the loss of parasitic activity and effectiveness of treatment is of the greatest importance. The promising method seems to be using of Positron Emission Tomography which enables to evaluate the glusose metabolism in hepatic lesion as glucose is the primary source of energy for endoparasitic helminths (Reuter et al., 1999) but this method is not performed routinely during the follow-up. Serological diagnosis is crucial not only in an early detection of infection, but also in long-term monitoring of patients after surgery and/or chemotherapy. Changes in levels of circulating IgG immune complexes, specific IgG1 and IgG4 subclasses of antibodies were found to reflect well the active parasitic lesions or their regression and death in several human metacestode infections (Short et al., 1990; Wen et al., 1995).

Since the first reported case of fatal human alveolar echinococcosis in former Czechoslovakia in 1977 (Šlais et al., 1979), for serological diagnosis of AE and CE the comercially available specific echinococcus antigens are used, which ensure reliable and comparable results. E. granulosus and E. multilocularis share many common antigens, but for the differential diagnosis the specific E. multilocularis antigens Em2+ and Em18 are recommended for diagnosis of human AE (Gottstein et al., 1989; Ito et al. 1995). In our study, the concentration of total IgG antibodies (expressed as antibody units in \%) to Em2+ antigen was in a high correlation with IgG to EmP antigen during the follow up of all patients. The highest IgG antibody levels at the time of diagnosis were detected in patients 1 and 2 and 4, where lesions were extensive. However, serum IgG and IgG4 activity had different patterns of activity during the follow-up. If extensive organ afflictions are present along with the inoperable localisation of the parasite, chemotherapy with high daily doses of albendazole or mebendazole is essential for many years or even life-long (WHO, 1996; Amman et al., 1994). Surgical removal of parasitic lesions was not indicated in these due to the advanced age of patients and presence of other chronic diseases. Chemotherapy with albendazole, or mebendazole was the only alternative and patients were on chemotherapy for more than two years. Initially both patients were on albendazole therapy, but in case 2 , the intolerance to the drug developed within three months. Therefore ABZ was replaced with mebendazole, which was better tolerated during the follow-up. Patients responded to the treatment by regression of the lesion in case 1 and stabilisation of parasitic lesion in case 2. Among the benzimidazole carbamates, albendazole is considered as the most effective, although various undesirable side effects were reported (Reuter et $a l ., 2000)$, what happened in our case 2. Mebendazole has a lower oral bioavailability and consequently lower plasma levels of the active metabolite than ABZ (Dayan, 2003), but the peak plasma levels of benzimidazoles in individual patients depend also on the metabolic capacity of liver parenchymal cells on cytochrome P450 (Rawden et al., 2000). This could markedly influence the drug's final the- 
rapeutical effect. Although in both our cases, numerous calcifications were observed on $\mathrm{CT}$, the rest of the parasitic tissue could provide sufficient antigenic stimulation. Matossian et al., 1992 believed that variations in IgG levels during the span of treatment indicate the persisting antigenic activity if cysts are present and diminished stimulus as the result of parasitic regression. Marked elevation of IgG4 antibodies in comparison with the other three cases were observed after a few years follow-up in patient 2 , indicating that lesions probably did not loose capacity to proliferate and relapse of disease therefore might occur. The high correlation between the response to surgery/ chemotherapy observed on $\mathrm{CT} /$ radiological examinations and changes in IgG4 levels were described by Wen et al. (1995) in a large group of patients with AE and authors concluded that reappearance of specific IgG4 antibodies is strong indication of recurrence. In cystic echinococcosis on Western Blot analysis using hydatid cyst fluid antigens, IgG4 binds to antigen B, the dominant diagnostic antigen secreted by active cysts (Wen \& Craig, 1994). In cured and "improved" patients with AE, binding of IgG4 to antigens with MW $21 \mathrm{kDa}$ and $17 \mathrm{kDa}$ decreased earlier than to other EmP antigens (Wen et al., 1995), however the role of IgG4 antibodies to various antigens in $\mathrm{AE}$ is not well understood.

The disease is only curable if AE is diagnosed in an early stage and the parasitic lesions are completely surgically removed (Eckert \& Amman, 1995). In case 3, the localisation of parasitic foci and the extent of the process allowed surgical removal of the whole parasitic mass. No metastatic cysts were infiltrated into other organs. Even in such cases, WHO recommended pre- and post-operative chemotherapy at least for 2 years (Eckert et al., 2001). In this patient, a rapid decline of IgG4 was observed, followed by the decrease of total IgG antibodies within two years of follow-up. Early diagnosis and the radical treatment approach proved to be successful in this case, as neither new foci, nor rise of specific antibodies was observed.

Another pattern of parasitic lesion was observed in case 4 . Calcifications in the foci were not detected indicating a possibly active cyst. Moreover, three new foci were formed inside this cyst containing purulent content. Necrosis of the pathological process can give rise to a large central cavity containing a gelatinous effusion with debris, bile and sometimes pus (Miguet et al., 1976). In contrast with the previous patients, who were on the same albendazole treatment schedule, neither regression nor stabilisation of cyst growth was observed on CT examination in case 4 . The predisposition for such disease progress could be associated with the formation of a new nutritive branch from a. hepatica com. which fed the foci. In addition, very high total $\mathrm{IgG}$ and $\mathrm{IgG} 4$ antibody titres persisted during the whole follow up indicating on continuous antigenic stimulation by live parasitic tissue forming et least part of the lesion. The real diagnostic difficulties of AE arise from primary hepatic neoplasms and metastases. US and CT patterns of such lesions can be quite similar to those described in $\mathrm{AE}$, including calcifications and biliary dilatations
(Stanley, 1983). Some diffuse or multiple hemangiomas have the same echogenic US pattern as AE, but on CT sequential enhancement properties are sufficiently characterristic (Didier et al., 1985). In the primary diagnosis of AE, US and CT scans are only supplementary. CT scan can better visualise lesions and newly formed foci, US is more suitable for visualising necrosis and abscesses (Reuter et al., 2001). Angiography can also provide invaluable information since in AE it shows rather specific images of arterial tapering and obstruction (Stanley, 1983; Hattingen et al., 1996). In our study the calcified foci found inside the main parasitic lesion were the only abnormality, which was detected in two cases ( 1 and 2 ) by the CT image technique. In other two cases $(3,4)$ where calcifications were absent, the parasitic cysts were expected to be active.

Based on the experiences with diagnostics and treatment of our four patients, we can conclude that in the absence of clinical signs, the infection was discovered by chance at US examination indicated for other causes. The finding was always suspected as malignant and the patient underwent the whole range of examinations including biopsy, which have not confirmed the diagnosis. Serological tests were performed subsequently to the histological examination of per-operative biopsy, while the procedure should have been in the reverse order. This would be more costeffective and less stressful to the patients. Therefore, it would be advisable prior to each laparotomy to take serum sample for serological examination for the presence of specific IgG and IgE antibodies and get more information about a patient's epidemiological history. Then during follow-up monitoring of total IgG antibody and IgG sub-classes, particularly IgG4 and IgG1 by ELISA and Western Blot seemed to be very useful for the assessment of disease prognosis. However, such complex approach is not applied in the routine serological diagnosis in Slovakia.

\section{Acknowledgment}

This work was suppored by Slovak Grant Agency VEGA no. $2 / 4179 / 26$.

\section{References}

AMmAN, R. W., ECKERT, J. (1996): Echinococcus. In WeInstock, J. V. (Ed): Parasitic diseases of the liver and intestines. Saunders, Philadelphia, pp 655 - 689

Amman, R. W., ILITSCh, A., MARINCEK, B., FreiburGHAUS, A. U. (1994): Effect of chemotherapy on the larval mass and the long - term course of alveolar echinococcosis. Swiss Echinococcosis Study Group. Hepatology, 19: $735-742$

Bresson-Hadni, S., Vuitton, D. A., Bartholomot, B., Godart, D., Meyer, J. P., Hrusovsky, S., Becker, M. C., Miguet, J. P. (2000): A 20-year history of alveolar echinococcosis in humans. Analysis of a series of 117 patients from eastern France. Eur. J. Gastroenterol. Hepatol., $12: 327-336$ 
CRAIG, P. (2003): Echinococcus multilocularis. Curr. Opin. Infect. Dis., 16: $437-444$

DAYAN, A. D. (2003): Albendazole, mebendazole and praziquantel. Review of non-clinical toxicity and pharmacokinetics. Acta Tropica., 86: 141 - 159

Didier, D., Weiler, S., Rohmer, P., Lassegue, A., DesChamps, J.-P., Vuitton, D., Miguet, J. P., Weill, F. (1985): Hepatic alveolar echinococcosis: Correlative US and CT study. Radiology; 154: 179 - 186

ECKERT, J., AMMAN, R. W. (1995): Clinical diagnosis and treatment of echinococcosis in human. In THOMPSON, R. C. A., LYMBERY, A. J., (Eds): Echinoccocus and hydatid disease. Wallingford: CAB International, pp 411 - 463

ECKERT, J., DEPLAZES, P. (1999): Alveolar echinococcosis in humans: the current situation in Central Europe and the need for countermeasures. Parasitol. Today, 15: 315 - 319 Eckert, J., Gottstein, B., Heath, D., LiU, F. J. (2001): Prevention of echinococcosis in humans and safety precations. In Eckert, J., Gemmel, M. A., Meslin, F. X., PAWLOWSKI, Z. S. (Eds): WHO/OIE Manual on Echinococcosis in humans and animals: a public health problem of global concern. Paris: WHO and OIE, pp $238-247$

Giraudoux, P., RaOUl, F., Bardonnet, K., Vuillaume, P., Tourneux, F., Cliquet, F., Dellatre, P., Vuitton, D.-A. (2001): Alveolar echinococcosis: characteristics of a possible emergence and new perspectives in epidemiosurveillance. Méd. Mal. Infect., 31: $247-256$

Gottstein, B., Tschudi, K., Eckert, J., AmMAnN, R. (1989): Em2-ELISA for the follow-up of alveolar echinococcosis after complete surgical resection of liver lesions. Trans. Royal Soc. Trop. Med. Hyg., 83: $389-393$

HATTINGEN, J., UERLINGS, A., JAUSSI, R. (1996): Alveoläre Echinokokkose der Leber mit Lymfogener pulmonaler und zerebraler Metastasierung. Forschr Rontgenstr, 1: 91 - 94 Ishizu, H., Uchino, J., SATO, N., AOKI, S., SuzUKI, K., KURIBAYASHI, H. (1997): Effect of albendazole on recurent and residual alveolar echinococcosis of the liver after surgery. Hepatology, 25: 528 - 531

Ito, A., Schantz, P. M., Wilson, J. F. (1995): Em18, a new serodiagnostic marker for differentiation of active and inactive cases in alveolar hydatid disease. A. J. Trop. Med. Hyg., 52: $41-44$

Kern, P., Bardonnet, K., Renner, E., Auer, H., PawLowski, Z., Amman, R. W., Vuiton, D. A., Kern, P. (2003): European Echinococcosis Registry: human alveolar echinococcosis, Europe, 1982 - 2000. Emer. Inf. Dis., 9: $343-349$

KinČeková, J., Auer, H., ReiterovÁ, K., DubinskÝ, P., SzILÁGYIOVÁ, M., LAUKO, L., ASPÖCK, H. (2001): The first case of autochthonous human alveolar echinococcosis in the Slovak Republic. Mitt. Österr. Ges. Tropenmed. Parasitol., 23: $33-38$

KinČeková, J., ReiterovÁ, K., Dubinský, P., SzILÁGYiovÁ, M., Johanes, R., GotTAs, M. (2002): A second case of autochthonous human alveolar echinococcosis in the Slovak Republic. Helminthologia, 39: 193 - 196
LucIUS, R., BILGER, B. (1995): Echinococcus multilocularis in Germany: Increased awarenes or spreading of a parasite? Parasitol. Today, 11: 430 - 434

Matossian, R. M., Awar, G. N., Radwan, H., Craig, P. S., Meshefedjian, G. A. (1992): Immune status during albendazole therapy for hydatidosis. Annals Trop. Med. Parasitol., 86: $67-75$

Miguet, J. P., Monange, C., Carbillet, J. P. (1976): L'échinococcose alvéolaire du foie. A propos de 20 cas observés en Franche-Comté. Etude anatomo-pathologique. Arch. Fr. Mal. App. Dig., 65: $23-32$

Rawden, H. C., KoKwaro, G. O., Ward, S. A., EdWARDS, G. (2000): Relative contribution of cytochromes P450 and flavin-containing monoxygenases to the metabolism of albendazole by human liver microsomes. $\mathrm{Br}$. J. Clin. Pharmamacol., 49: 313 - 322

Reuter, S., Jensen, B., Buttenschoen, K., Kratzer, W., KERN, P. (2000): Benzimidazoles in the treatment of alveolar echinococcosis: a comparative study and review of the literature. J. Antimicrob. Chemother., 46: 451 - 456

Reuter, S., Nussle, K., Kolokythas, O., Hang, U., RieBER, A., Kern, P., Kratzer, W. (2001): Alveolar liver echinococcosis: A comparative study of three imaging techniques. Infection, 29: 119 - 125

Reuter, S., Schirrmeister, H., Kratzer, W., DreWeck, C., Reske, S. N., Kern, P. (1999): Pericystic metabolic activity in alveolar echinococcosis: Assessment and follow-up by positron emission tomography. Clin. Inf. Dis., 29: $1157-1163$

Rigano, R., Profumo, E., Ioppolo, S., Notargiacomo, S., Ortona, E., Teggi, A., Siracusano, A. (1995): Immunological markers indicating the effectiveness of pharmacological treatment in human hydatid disease. Clin. Exp. Immunol., 102: 281 - 285

Short, J. A., Heiner, D. C., HsiaO, R. L., Andersen, F. L. (1990): Immunoglobulin E and G4 antibodies in cysticercosis. J. Clin. Microbiol., 28: 1635 - 1639

STANLEY, R. J. (1983): Liver and biliary tract. In LEE, J. K. T., Sagel, S. S., Stanley, R. J. (Eds): Computed Body Tomography. New York, Raven Press, pp167 - 211

Šlais, J., MAdle, A., VANKA, K., JelíNEK, F., ČERNIK, M., PRŮCHOVA, M., JINDRA, J. (1979): Alveolar hydatidosis (echinococcosis) diagnosis by liver puncture biopsy. Čas. lék. čes., 15: $472-476$

Wen, H., Bresson-Hadni, S., Vuiton, D. A., Lenys, D., YANG, B. M., Ding, Z. X., CRAIG, P. S. (1995): Analysis of immunoglobulin $\mathrm{G}$ subclass in the serum antibody response of alveolar echinococcosis patients after surgical treatment and chemotherapy as an aid to assessing the outcome. Trans. Royal Soc. Trop. Med. Hyg., 89: $692-697$

Wen, H., Craig, P. S. (1994): Immunoglobulin G subclass responses in human cystic and alveolar echinococcosis. Annals Trop. Med. Hyg., 51: $741-748$

World HeAlth ORGanisation (1996): Guidelines for treatment of cystic and alveolar echinococcosis in humans. Bulletin of the World Health Organisation, 74: 231 - 242 